\title{
Ciencia y política en tiempos de guerra fría: un examen psicológico de niños españoles en el exilio*
}

\section{Science and politics in times of the Cold War: A psychological examination of Spanish children in exile}

Recibido: enero 14 de 2014 | Revisado: febrero 18 de 2014 | Aceptado: julio 17 de 2014

\author{
ANNETTE MÜLBERGER ** \\ Universitat Autònoma de Barcelona, España
}

doi:10.11144/Javeriana.upsy13-5.cptg

Para citar este artículo: Mülberger, A. (2014). Ciencia y política en tiempos de guerra fría: un examen psicológico de niños españoles en el exilio. Universitas Psychologica, 13(5), 1941-1953. http://dx.doi. org/10.11144/Javeriana.upsy13-5.cptg

La autora agradece la financiación de la investigación por parte del Ministerio de Economía y Competitividad (HAR2009-11342/HIST)

** CEHIC/Dept. Psicologia Básica, Evolutiva y de la Educación, Facultad de Psicología; Universitat Autònoma de Barcelona, E-08193 Bellaterra (Cerdanyola), España.E-mail: annette.mulberger@uab.cat

\begin{abstract}
A B S T R A C T
During the Second World War, physicians, psychiatrists, social workers, and psychologists developed a growing interest in studying the effect war had on the bodies and minds of children. Many of the observations were carried out in the 1940s in France, Great Britain, and the United States. With respect to the Spanish youth, no such interest related to the Civil War is known. The present article deals with a psychological study undertaken towards the end of the 1940s in France by a Spanish physician (named A. Piñar) with exiled children and teenagers, a study ignored up to now. The physician aimed at knowing what memories the children had from their experiences of the Civil War and the World War II, as well as evaluating the psychological consequences of these experiences. The study constitutes one of the few examples of a research exposing in a synthetic and clear way the emotional state of the Spanish youth at that time. It is important to situate the study in its scientific and historical context, with a particular focus on the political interests of the author. The physician called for medical and humanitarian attention to young immigrants. However, the historical moment was rather inconvenient for this, due to the new political situation marked by the Cold War.

Keyworks

Spain, exile, trauma, child psychology, testing
\end{abstract}

\section{RES U MEN}

A lo largo de la Segunda Guerra Mundial hubo una preocupación creciente por parte de médicos, psiquiatras, trabajadores sociales y psicólogos por el efecto que podía llegar a tener la guerra en los niños, tanto a nivel orgánico como psíquico. Gran parte de los estudios fueron llevados a cabo en Francia, Gran Bretaña y Estados Unidos, en la década de 1940. Respecto a la población infantil española, no se conocen apenas estudios realizados durante o poco después de la Guerra Civil. El presente artículo trata de una investigación psicológica poco conocida, llevada a cabo a finales de los años de 1940. En ella, un médico examinó niños y jóvenes españoles que vivían en Francia. Quería comprobar los recuerdos que tenían acerca de la Guerra Civil, el exilio y la Segunda Guerra Mundial, así como evaluar hasta qué punto estas experiencias dejaron secuelas psicológicas en los jóvenes. Se trata de una de las pocas investigaciones que muestran, de una forma sintética y clara, cómo estaban anímicamente algunos niños españoles en aquellos años. En el presente artículo expongo, asimismo, el contexto científico e histórico en el que se sitúa dicha investigación. A través de ello quedan claras las intenciones políticas del autor, quién reclamaba una mayor atención médica y humanitaria para los jóvenes exiliados. Sin embargo, el momento histórico era poco propicio. La nueva situación política de la Guerra Fría afectaba el trato que recibían los exiliados en Francia.

Palabras clave

España; exilio; trauma; psicología infantil; test 
"La guerre a créé presque expérimentalement des conditions qui ont fait naître et se développer selon la loi du grand nombre des symptômes intellectuels ou affectifs que nous rencontrions avant la guerre de façon seulement sporadique» (Heuyer, 1948, p. 3)

\section{Introducción}

Desde finales del siglo XIX, la vida infantil había sido objeto de hondas reformas higiénicas, pedagógicas y sociales. En 1900, la famosa pedagoga sueca Ellen Key había prometido para el siglo XX "el siglo de los niños", captando con tal eslogan un sentir de una época. En España, también se asentó, poco a poco, esta nueva mentalidad, siguiendo las recomendaciones realizadas en el marco de los congresos internacionales (Perdiguero, 2004; Rollet, 2001).

La Sociedad Española de Higiene, que comenzó sus andares a partir de la década de 1880, impulsó la medicina social con el objetivo de acrecentar el bienestar de la población y velar por la protección del débil. Perdiguero y Robles (2004) han mostrado cómo la preocupación por el niño formaba parte del proyecto reformador (regenerador) y moralizador más amplio, perseguido por la burguesía de la época. Gracias a tales iniciativas y el liderazgo del pediatra Manuel Tolosa Latour (1857-1919), se aprobó en 1904 la Ley de Protección de la Infancia, con la que se pretendía mejorar la situación de los menores de 10 años a través de un mayor control de la lactancia mercenaria y de instituciones como casas de cuna, escuelas y asilos (Barona, 2004).

Sin embargo, la falta de apoyo político y financiero lentificó el proceso e impidió una intervención efectiva inmediata. Desde un principio, el modelo de protección instaurado en España carecía de un compromiso del Estado para su financiación. Por ello, durante los años posteriores a la aprobación de la ley de protección, el cuidado de los niños abandonados seguía en manos de la caridad y la filantropía (Barona, 2004). Mientras tanto, en revistas como Pro Infantia, circulaba cada vez más información sobre la falta de protección de la infancia y, especialmente, sobre el problema de la mendicidad infantil
(Barona, 2004). De esta forma, se puede constatar que con el comienzo del siglo XX la preocupación por el bienestar del niño se instauró poco a poco en la sociedad española. Sin embargo, un cambio real en la situación de los sectores más necesitados no se dejó notar hasta los años veinte.

Mientras tanto, las guerras impondrían nuevos retos y dificultades a este proceso. El estallido de la Primera Guerra Mundial (1914-1918), la Guerra Civil española (1936-1939) y la Segunda Guerra Mundial (1939-1945) tuvieron consecuencias nefastas para la población y también para el sector infantil. Incrementaron todo tipo de problemas como la orfandad, la falta de alimento e higiene, las experiencias traumáticas, la explotación laboral y el éxodo. En las naciones sumergidas en un estado de guerra, las posibles secuelas psicológicas que podrían sufrir los niños no constituían una preocupación central ni inmediata, dado que la movilización militar y la ayuda humanitaria para suplir las necesidades más básicas de la población fueron consideradas prioritarias.

Aun así, la psicología infantil en tiempos de guerra estuvo presente. A lo largo de la Segunda Guerra Mundial hubo una preocupación creciente por parte de médicos, psiquiatras, trabajadores sociales y psicólogos por el efecto que podía llegar a tener la guerra en los niños, tanto a nivel orgánico como psíquico. Gran parte de los estudios fueron llevados a cabo en Francia, Gran Bretaña y Estados Unidos, en la década de 1940. Apenas existen investigaciones psicológicas con la población infantil española, realizadas durante o poco después de la Guerra Civil.

A lo largo de los últimos años ha habido un creciente interés por conocer las inquietudes y vivencias de los niños españoles exiliados, analizadas a partir de dibujos (Duroux, Baudon, \& Gaboardi, 2006; Gallardo \& Gallardo, 2011), cartas (Sierra, 2009) y testimonios posteriores (Aldecoa, 1983; Alted, 1996; Benjamin, 2007; Bravo et al. 1993; Martín \& Carvajal, 2002; Pàmies, 1977). En la literatura sobre el exilio se pueden encontrar diagnósticos psicológicos realizado post-hoc, en base a recuerdos personales o documentos primarios, una tarea que siempre resulta difícil y un tanto arriesgada. 
A continuación, voy a destacar una contribución científica muy original, realizada a finales de los cuarenta con niños españoles exiliados en Francia (Piñar, 1949). El estudio trata de evaluar las secuelas psicológicas de la Guerra Civil y la Segunda Guerra Mundial. Estaba guiado por preguntas como: ¿qué sabían realmente los niños y jóvenes acerca de lo que pasó? y icómo les afectó intelectual y anímicamente? Aunque la publicación de la que trata el presente trabajo no contiene un gran número de testimonios detallados, la información que aporta resulta clave y cubre un importante vacío historiográfico en relación con el examen psicológico realizado a la población exiliada. Estamos ante una de las pocas investigaciones que muestran, de una forma sintética y clara, cómo estaban psicológicamente algunos niños españoles a finales de los años cuarenta.

A través de mi contribución histórica, quiero mostrar cómo el interés por la mente infantil queda reflejado en esta investigación. Para contextualizarla, la compararé con otros estudios psicológicos publicados en la misma década. Más allá de la simple exposición de una investigación desconocida, el objetivo principal de mi trabajo es identificar las intenciones políticas del autor y mostrar la situación histórica en la que se encontraban tanto los niños como el científico que los estudia. Para captar el alcance de estas intenciones, será necesario tener en cuenta el proyecto del hospital Varsovia, así como, de forma más general, el exilio republicano y las tensiones políticas de la Guerra Fría en Europa.

\section{Estudios psicológicos del niño en tiempos de guerra y posguerra}

Los estudios psicológicos y psiquiátricos sobre las consecuencias de la Primera Guerra Mundial fueron todavía escasos, y algunos informes de la época destacaban simplemente que la población consiguió adaptarse bastante bien a la nueva situación bélica (Kimmins, 1915). Asimismo, hay pocos trabajos que hacen referencia al efecto de la Guerra Civil española. Destaca la labor de Mercedes Rodrigo al frente del Instituto Psicotécnico y su colaboración en la evacuación de civiles en Madrid (Herrero,
2003). En relación con los bombardeos aéreos, el escritor inglés John Langdon-Davies (1938) describía las reacciones de pánico de la población, mientras el psicólogo y psiquiatra catalán Emilio Mira y López (1939) consideraba que los ataques no tuvieron un efecto psíquico tan severo en la población como sí lo tuvo el hambre. Señaló, además, que los niños se mostraron, en general, bastante más tranquilos que los adultos.

Con el comienzo de la Segunda Guerra Mundial, la preocupación por el bienestar psicológico de los niños incrementó notablemente. El balance de Jersild y Meigs (1943) que se basa en la revisión de 141 investigaciones permite distinguir algunos cambios en la orientación de los trabajos. La apreciación de un afrontamiento exitoso y valiente de la situación por parte de la población, presente en las publicaciones relativas a la Primera Guerra Mundial, cambió en la literatura posterior sobre las reacciones ante la Segunda Guerra Mundial. Los autores (en general médicos, pedagogos, asistentes sociales y psicólogos) emplearon ahora una retórica más alarmante, refiriéndose a las tensiones internas y traumas a largo plazo, producidas por la situación bélica y sus consecuencias (véanse, por ejemplo, Gerard, 1943; Heuyer, 1948; Mac Donald, 1943; Mercier \& Despert, 1943; entre otros).

En general, los investigadores trabajaban mediante un enfoque clínico y psicológico, a dos niveles. Comprobaban, en primer lugar, el conocimiento y grado de concienciación que tenían los niños y jóvenes del conflicto (Kimmins, 1915, Jersild y Meigs, 1943). En un segundo nivel, querían apreciar los efectos psicológicos de la nueva situación. Para ello, aparte de tomar medidas antropométricas, solían examinar mediante entrevistas, test psicológicos o cuestionarios las capacidades intelectuales y el estado afectivo de los jóvenes (Despert, 1944; Jersild \& Meigs, 1943; Stückelberger, 1943; Young, 1947).

Así, una investigación realizada por Preston (1942) mostró que mientras los niños de 9 años de Nueva York solo disponían de información suficiente para responder correctamente un $60 \%$ de preguntas sobre la guerra, en un grupo de 14 años el porcentaje subió hasta casi un $80 \%$. Con ello confirmó la idea de Kimmins (1915) de que niños 
preadolescentes suelen tener solo una idea muy vaga de los acontecimientos de la guerra, ignorando gran parte de las dimensiones políticas del conflicto.

Habitualmente, el niño es menos capaz de expresar en palabras sus pensamientos y miedos. Aun así, reacciona con fuertes sentimientos ante las circunstancias que amenazan con interferir en su vida personal. En relación con los efectos emocionales, algunos trabajos norteamericanos evidenciaron que más de un tercio de los niños solían tener sueños relacionados con la guerra y que muchos habían experimentado miedo, tristeza o disgusto al ver, por ejemplo, películas mostrando bombardeos o escenas de guerra (véase el trabajo de Gastwirth \& Silverblatt de 1942 comentado por Jersild \& Meigs, 1943, p. 546). En general, los estudios destacaron la evacuación o el exilio y, sobre todo, la separación forzosa entre madre e hijo/a, como causa principal de posibles problemas psicológicos en el niño. Signos comunes de estos problemas serían la incontinencia, un estado de nerviosismo general y, en casos excepcionales, incluso síntomas neuróticos y problemas en el desarrollo cognitivo (Young, 1947). Estas alteraciones se expresaban, a menudo, a través de reacciones de miedo o depresión, problemas de sueño, irritabilidad general, trastornos alimenticios y, en niños más pequeños, llanto frecuente (Despert, 1944).

Con los años, los trabajos se centraron cada vez más en los aspectos emocionales y los posibles síntomas neuróticos producidos por la guerra. Un buen ejemplo de una investigación psicológica es la que llevaron a cabo Rautman y Brower (1951), donde analizaron las respuestas dadas por una amplia muestra de escolares ante 10 imágenes seleccionadas del Thematic Apperception Test de Murray. Los resultados obtenidos con 536 alumnos en 1943 y con otra muestra de 468 niños en 1950 mostraron un descenso significativo de la preocupación por el tema de la guerra. Cuando se les pidió a los niños que explicaran una historia basada en cada imagen, se pudo observar que también había aumentado el número de historias con final feliz.

Como ha señalado recientemente Zahra (2009), en el abordaje de los problemas psicológicos de la población infantil durante de la Segunda Guerra
Mundial, se nota un creciente dominio de un enfoque individualista y psicoanalítico ${ }^{1}$. Los estudios apuntan, en general, hacia el binomio madre-hijo como eje fundamental para garantizar un afrontamiento eficaz por parte del niño (Gerard, 1943). Con ello, cargan a la mujer con la responsabilidad del bienestar físico y psíquico del infante y utilizan el núcleo familiar como elemento básico para contrarrestar la falta o la ineficacia de las instituciones en suplir las necesidades psicológicas. Un ejemplo citado frecuentemente en este contexto es el trabajo de Anna Freud y Dorothy Burlingham. A partir de sus experiencias en las guarderías de guerra, The Hampstead war nurseries (véanse, Freud \& Burlingham, 1942), las autoras concluyeron que especialmente desde el segundo año de vida, la separación de la madre resulta necesariamente traumática y debía ser evitada a toda costa (Midgley, 2007). En casos en los que no es posible impedirlo, la presencia de una madre substituta puede ser eficaz para aliviar el sufrimiento del niño.

Sin duda, el efecto de la guerra es distinto si el país es solo bombardeado o si, además, es invadido por fuerzas enemigas (Despert, 1944). Este fue el caso de Francia, donde los médicos parisinos constataron un efecto más dramático de las circunstancias políticas y donde, en general, la atención médica fue extremamente difícil, debido a los impedimentos para poder desplazarse y la escasez general de comida, vitaminas y medicinas. Así, Mercier y Despert (1943) presentaron una serie de casos de niños que dieron claras muestras de sufrimiento psíquico ante la nueva situación. En la primera reunión de "Semaines Internationales pour l'Étude de l'Enfance Victime de la Guerre" (SEPEG) celebrada en Zúrich en 1945, G. Heuyer (1948) habló de la "psicosis colectiva" producida por las evacuaciones forzosas y los bombardeos. Según el médico, el éxodo y la marcha del padre al frente propiciaron una alteración en la actitud moral de la mujer, que luego tendría consecuencias graves en el carácter del infante o el adolescente. De esta forma,

1 Un ejemplo muy claro es el trabajo de Ross (1943) y Gerard (1943), pero muchos otros autores también utilizaban una terminología psicoanalítica y psicopatológica en sus estudios. 
insistió en las consecuencias nefastas de la ausencia de la figura paterna para el desarrollo del joven: "Hemos visto que los trastornos constitucionales del carácter se agravaron en los desequilibrados, privados de la autoridad paterna, de la vigilancia y del control necesario" (Heuyer, 1948, p. 6; la traducción es mía). Según Heuyer, esta situación y la incapacidad de la madre de proporcionar un contexto educativo y psicológico adecuado, causaría un incremento en el número de niños "abandonados moralmente" y llevaría a muchos jóvenes hacia la delincuencia. Mientras en 1938 el número de juicios por criminalidad infantil en Francia estuvo alrededor de 10.000, en 1944 esta cifra había aumentado a 44.000 (Heuyer, 1948) ${ }^{2}$.

La literatura sobre las consecuencias psicológicas de la guerra enfatiza el ambientalismo en la medida en que mostraba los efectos que tiene la situación personal y social en el psiquismo individual, aunque muchos autores planteaban una interacción entre el ambiente y las características constitucionales de la personalidad. Tal enfoque fomentó la diferenciación individual y el estudio de casos concretos (véase, por ejemplo, Young, 1945). Aunque todos coincidieron en que existe una influencia psicológica de la situación política, las opiniones divergieron en el momento de apreciar el peso y la clasificación de la misma. A través de un examen de publicaciones británicas y estadounidenses tanto Despert (1944) como Jersild y Meigs (1943) concluyeron que en general los niños superaron bien la situación amenazante del conflicto. Las reacciones a los estímulos potencialmente traumáticos habían sido transitorias y habían afectado principalmente a niños con una inestabilidad emocional previa.

Otros autores (generalmente de lengua francesa) insistieron en las consecuencias graves que la guerra tuvo y tendría a largo plazo en la personalidad de los jóvenes afectados, provocando cuadros de ansiedad también en niños sin predisposición o problemas previos (Brosse, 1949; Heuyer, 1948;

2 En una muestra de 300 jóvenes delincuentes, solo el $15 \%$ provenía de un ambiente familiar normal y completo (Heuyer, 1948). El incremento de la delincuencia infantil a partir de 1943 fue observado a escala internacional (Brosse, 1948; Despert, 1944; Mac Donald, 1943; Jersild \& Meigs, 1943).
Jouhy \& Shentoub, 1949; Mercier \& Despert, 1943; Wolf-Machoel, 1945). En esta línea, el holandés Wolf-Machoel, colaborador de Bovet en el Instituto J. J. Rousseau de Ginebra, mantuvo una posición extrema, al plantear una patología psicológica específica para las víctimas de la guerra. Así, habló de la psicosis de los internados (psychose des internés) y la psicosis de los desplazados (psychose des déracinés).

A pesar de las diferencias, los investigadores coincidieron en señalar que:

a) Las reacciones de la población infantil ante situaciones de amenaza como bombardeos no fueron tan severos como en los casos de evacuación (Bodman, 1944). Dependían, sobre todo, de las emociones exhibidas por los mayores que les rodeaban (Beverly, 1943). Por ello, muchos recomendaban evitar a toda costa las evacuaciones de la población.

b) En las mediciones con test, el nivel de inteligencia de la población infantil desplazada se mostraba como normal (a pesar de deficiencias en la formación escolar reglada).

c) Las situaciones de desplazamiento e internamiento forzoso, así como la desintegración familiar, tuvieron un efecto negativo en la actitud moral (carácter o personalidad) del joven, que solía comenzar a mentir, a mostrar una actitud desobediente ante la autoridad, además de una precocidad psíquica y sexual.

Finalmente, a medida que se alargaba la guerra, los investigadores se preocupaban cada vez más por el incremento de la delincuencia infantil y juvenil y las posibles secuelas psicológicas de la guerra a largo plazo.

\section{El estudio psicológico de niños españoles exiliados en Francia}

\section{El investigador y su muestra}

Cuatro años después de acabar la Segunda Guerra Mundial, era posible plantear la comprobación acerca de la existencia de secuelas a largo plazo. Esto es lo que hizo un médico español exiliado en Francia. Disponemos de poca información biográfica sobre el autor. Su nombre fue Antonio-Jesús Piñar Jiménez (o Giménez), nacido en 1903 en Málaga (Archidona) 
(Ferrán Sánchez, 2008). Se había licenciado en medicina en 1924 por la Universidad Central de Madrid (Guerra, 2003). Durante la Guerra Civil había sido capitán al mando de Sanidad del Ejército Republicano; después de la guerra se exilió como muchos otros científicos (Barona, 2010; Giral, 1994; Zarzoso \& Martínez Vidal, 2011) y antes de terminar la Segunda Guerra Mundial fue internado en campos de concentración franceses (Guerra, 2003). Una vez acabada la guerra, pudo trabajar de médico, asistiendo a emigrantes españoles en Lodève (Hérault) y en el Hospital de Varsovia. Dicho hospital fue creado en 1944 durante las operaciones de reconquista lanzadas desde el Valle de Arán en contra del régimen de Franco. Servía, en primer lugar, para atender a los guerrilleros antifascistas heridos ${ }^{3}$, aunque en seguida atendía también a la población exiliada en general (Martínez Vidal, 2010). Contaba con pocos medios y aprovechaba las ayudas recibidas por parte de organizaciones humanitarias norteamericanas y europeas (especialmente por parte del Joint AntiFascist Refugee Committee y el Unitarian Service Committee). Situado en un castillo abandonado por los alemanes en las afueras de Toulouse (en el barrio de Saint-Cyprien donde se concentraba gran parte de los refugiados españoles), recibió el nombre por la calle en la que se encontraba. Hasta 1950 y gracias a la Ley Billoux los médicos españoles exiliados, aunque no tenían el título reconocido en Francia, podían trabajar en el hospital, atendiendo a compatriotas (Martínez Vidal, 2010).

En su artículo, Piñar informa que examinó 81 niños y jóvenes refugiados españoles entre siete y diecinueve años. No dice dónde realizó el estudio, pero es probable que fueran los niños acogidos en la Maison des Enfants de Saint-Goin ${ }^{4}$. Se trataba de una casa de colonias que estaba situada en los Pirineos Atlánticos (Alta Navarra) y que acogía niños para prevenir la tuberculosis. Sostenida por el Unitarian

3 Se trataba de miembros de agrupaciones como el FFI (Fuerzas Francesas del Interior), la AGE (Agrupación de Guerrilleros Españoles) y el MOI (Main d'Oevre Immigrée) (véase Guixé, 2010).

4 Veremos más adelante que Piñar tenía razones para ocultar su paradero. La hipótesis de la ubicación fue facilitada por Àlvar Martínez Vidal en una conversación privada.
Service Committee, ofrecía un lugar para estancias cortas, de unas semanas, para niños provenientes de un entorno familiar con enfermos tuberculosos. Posiblemente a lo largo del periodo de funcionamiento (aproximadamente entre 1948 y 1952) ofreciera también un lugar para alojar a niños huérfanos españoles.

Piñar alertaba sobre la diferencia que hay entre las experiencias de guerra vividas por niños en Estados Unidos o Inglaterra respecto a la de los niños españoles refugiados en Francia. Estos últimos tuvieron que superar la experiencia de dos guerras, la Guerra Civil y la Segunda Guerra Mundial. Además, sufrían el destino de ser exiliados, lo que para muchos significaba haber tenido que pasar un tiempo en campos de refugiados. La situación se agravó con la ocupación de Francia por la Alemania nazi. Finalmente, en tiempos de Guerra Fría, tuvieron que vivir con el estigma de ser considerados "hijos de rojos". Así, observaba el médico, estos niños "[S]on, por lo tanto, buenos sujetos para investigar los cambios y trastornos psicológicos determinados por los factores bélicos" (Piñar, 1949, p. 1).

\section{Los resultados de los test psicológicos}

En la investigación, Piñar registró, en primer lugar, variables como la edad (biológica), el cociente intelectual, la edad escolar, el perfil psicológico y un diagnóstico caracterológico, y en segundo lugar, preguntaba por los sueños. Utilizó un cuestionario de 32 preguntas para conocer los recuerdos de las guerras que cada niño tenía (comentaré los resultados de esta parte en el siguiente apartado).

Para conocer el grado de madurez intelectual, el perfil psicológico y el carácter de los jóvenes, Piñar (1949) combinaba diferentes instrumentos psicológicos: test estandarizados y comercializados como el Binet-Simon, el Terman y el Rorschach, conjuntamente con un cuestionario diseñado específicamente para investigar las secuelas psicológicas. La combinación de pruebas reactivas y proyectivas (estandarizadas y específicas) puede resultar sorprendente, pero corresponde a la idea, muy común en la época, de que es necesario emplear una batería de diferentes instrumentos psicológicos para medir y captar los diversos aspectos de la personalidad hu- 
mana. Nos encontramos en un período de expansión y universalización de los test psicométricos y de los psicodiagnósticos. Había expertos, como por ejemplo, Edouard Claparède y Emilio Mira y López que combinaban test intelectuales de tipo diferencial con pruebas proyectivas psicoanalíticas (Kirchner, 1974).

A partir de la aplicación de las pruebas de inteligencia, Piñar constató una variabilidad del coeficiente entre 0.62 y 1.33 , con una media en torno al 1 , lo que mostraría que los jóvenes exiliados tenían un grado de inteligencia aceptable. Pero la edad escolar, es decir, el avance en la escolaridad era en el $90 \%$ de los casos retrasado en más de un año. Sabiendo que los niños tuvieron que interrumpir sus estudios en la guerra y que, una vez en Francia, tuvieron problemas para asistir a una escuela (además de problemas lingüísticos), este retraso resulta comprensible (véase Fondation Nationale des Sciences Politiques, 1999). Éstos resultados concuerdan plenamente con los de otros investigadores como Wolf-Machoel (1945), quien, tras examinar niños refugiados de diversos países, comprobó el nivel de inteligencia normal de la muestra, combinado con un fuerte retraso escolar.

En general, el perfil psicológico de los jóvenes exiliados exponía puntos fuertes y puntos débiles. Entre los primeros, destacaba una memoria superior a la media para las formas y una excelente coordinación motriz. Entre los segundos, se señalaba la falta de "atentividad", es decir, dificultades para mantener la atención y una memoria auditiva para la música y las palabras ligeramente inferior a la media (Piñar, 1949). Este dato también coincide con los de Wolf-Machoel (1945), quién a través del test de Piéron encontró problemas de atención en los jóvenes refugiados, al compararlos con una muestra de niños suizos.

En cuanto al carácter, Piñar encontró una falta de fijación afectiva en el $40 \%$ de los niños, un dato obtenido a través de las reacciones de los sujetos a las manchas del test de Rorschach. Este porcentaje es relativamente alto y, según el médico, está ligado al trauma de la guerra vivido por los niños. Asimismo, investigadores británicos habían observado con anterioridad algunos efectos psicológicos de las experiencias de niños con bombardeos, registrados con el test de Rorschach (Alcock, 1941).

\section{La encuesta: los recuerdos de la guerra y su rastro psicológico}

Para conocer más de cerca qué tanto y cómo recuerdan sus vivencias los niños exiliados, los sometió, en una segunda fase de su investigación, a un cuestionario bastante exhaustivo. Piñar (1949) planteó 32 preguntas abiertas sobre las experiencias vividas durante las dos guerras (Guerra Civil Española y Segunda Guerra Mundial). De esta manera, el autor estudiaba el recuerdo de acontecimientos que tuvieron lugar cuando estos jóvenes tenían entre 1 y 13 años. En gran parte, sus preguntas fueron informativas, pensadas para apreciar el grado de conocimiento que tenían de los hechos. Preguntó, por ejemplo: icuándo comenzó la guerra? y isupuso una interrupción para tus estudios?

Otras preguntas trataban de evaluar de qué manera los hechos históricos afectaron personalmente al niño: ihubo muertos o heridos entre tus parientes cercanos?, ihas presenciado alguna vez algún combate o viste alguna ejecución? o ifuiste deportado alguna vez? De forma más directa intentaba apreciar la actitud del niño o joven respecto a la guerra con preguntas como: ite gustaría que hubiese otra guerra? y isueñas con la guerra? Es una pena que en su informe no consten las respuestas transcritas de todos los entrevistados. Piñar ofrece un resumen general de todos los resultados y añadió solo las respuestas concretas de cuatro niños españoles de 11 a 16 años, a modo de ejemplo.

En su estudio, Piñar (1949) encontró que mientras los niños franceses parecían tener pocos recuerdos de la guerra y no se mostraban preocupados por el tema, los niños españoles estaban todavía muy afectados (Mülberger, 2013). De los resultados presentados por el informe destacan los siguientes aspectos:

1. Todos los niños vivían en un entorno familiar disociado, un $40 \%$ de ellos eran huérfanos de padre.

2. En general, los niños no tenían recuerdos de la Guerra Civil Española.

3. Más de la mitad de los encuestados (64\%) mantenían algún recuerdo de bombardeos de la Segunda Guerra Mundial y aproximadamente la misma cantidad de jóvenes sufría todavía pesadillas relacionadas con la guerra. Comparado con 
estudios realizados en Estados Unidos unos años antes, esto constituye un porcentaje relativamente alto (Jersild \& Meigs, 1943).

4. Una gran parte de los niños (concretamente el $75 \%$ ) presenció la llegada de los alemanes y por tanto vivió de forma muy consciente la ocupación militar de Francia. El mismo porcentaje de jóvenes $(73 \%)$ recordaba la victoria y las fiestas para celebrarla. En unas encuestas realizadas a 1137 niños franceses, Lézine (1948) había encontrado asimismo impresiones profundas que estas experiencias habían dejado en la memoria. Además obtuvo dibujos y redacciones que le permitieron estudiar más en profundidad y de forma cualitativa tales recuerdos.

5. Más de la mitad (un $60 \%$ ) estuvo alguna vez en un campo de concentración, refugio o colonia infantil, un dato que corrobora las observaciones de otros historiadores (Marqués, 1993).

6. Casi todos los niños (un $90 \%$ ) denotaban un retraso escolar debido a las circunstancias de la guerra y el exilio. Como se ha visto, esto fue una característica muy habitual en la población infantil de la posguerra, especialmente entre los desplazados.

7. Todos los niños habían vivido, y en ocasiones todavía estaban teniendo, dificultades para cubrir las necesidades básicas (falta de alimentación, alojamiento o vestimenta).

Piñar se preocupaba por el rastro dejado por la guerra. Un rastro que consistía básicamente en un recuerdo de bombardeos de la Segunda Guerra Mundial y las dificultades de la vida cotidiana en el exilio. El informe termina con una recomendación concreta: los niños españoles necesitan ayuda humanitaria y psicológica. Pero el llamamiento llegó en un momento histórico poco propicio.

\section{Los niños españoles, las guerras y el exilio}

Los niños de la investigación de Piñar fueron supervivientes. En total, se estima que más de 400.000 niños habían perdido la vida durante la Guerra Civil (Sierra, 2009). De los niños que sobrevivieron, más de 30.000 tuvieron que emigrar. Desde noviembre de 1936 se creó un Comité d'Accueil aux Enfants de l'Espagne que actuaba en coordinación con la República Española para organizar el desplazamiento de los jóvenes (Martín \& Carvajal, 2002).

Los testimonios de la época (Aldecoa, 1983; Alted, 1996; Martín \& Carvajal, 2002; Pàmies, 1977) muestran que pasar la frontera durante la posguerra era costoso y arriesgado. Un niño de catorce años, huérfano (de padre y madre), estudiado por Piñar (1949), recordaba haber emigrado a Francia. Poco después había sido deportado de nuevo a España por lo que en seguida volvió por segunda vez a cruzar la frontera de los Pirineos para llegar a Francia. Aunque no da más detalles, podemos suponer que los tres viajes resultarían problemáticos y retrasaron su escolarización hasta los 9 años de edad.

Cuando en 1939 llegaban cada vez más españoles a tierras francesas, Francia se veía desbordada. La actitud de la población hacia los refugiados fue en muchos casos de rechazo, por considerarlos revolucionarios y miserables (Alted \& Domergue, 2003). Los españoles fueron retenidos y hacinados en unos campos de refugiados, estrictamente vigilados (Cuesta \& Bermejo, 1996; Martín \& Carvajal, 2002; Rafaneau-Boj, 2010). Durante algún tiempo, el movimiento antifranquista de la resistencia estuvo vivo. Pero tras varios fracasos, los planes militares de la guerrilla se vieron truncados con la ocupación alemana. Por un lado, esto significó el envío de españoles a campos de concentración del norte ${ }^{5}$, y por otro, guerrilleros franceses y españoles se unían en la lucha antifascista de resistencia (los denominados "maquis") (Nieto, 2007). Entre los refugiados se hablaba e, incluso, se ayudaba a los guerrilleros escondidos en los Pirineos (Bravo et al., 1993). El cuestionario de Piñar muestra que el 28 \% de los jóvenes entrevistados por él sabían detalles acerca de las actividades de los maquis (Piñar, 1949).

La lucha unida entre españoles exiliados y franceses para liberar a Francia mejoró momentáneamente las relaciones entre los dos colectivos. $\mathrm{La}$

5 Según Marques (1993) el 50 \% de los niños internados en los campos de concentración eran españoles. Sobre la organización y la vida en los campos véase los testimonios recogidos por Ginestá (2010). 
gran celebración de la liberación de Francia era recordada por los niños y jóvenes entrevistados por Piñar como un momento de unión y felicidad, notándose cierto cambio de actitud de los franceses respecto a los emigrados del sur.

Pronto las relaciones volverían a hacerse difíciles. En el contexto emergente de la Guerra Fría la simpatía hacia los exiliados (entre los que hubo republicanos y comunistas) decrecía ante los ojos de la opinión pública. Ahora había un interés más marcado por restablecer las relaciones comerciales con la España de Franco y hacer frente común contra el comunismo soviético (Cervera, 2007; Glondys, 2012; Guixé, 2002). Instigada por las autoridades franquistas, la policía francesa llevó a cabo de madrugada (en 1950) la "operación BoléroPaprika”. Numerosos refugiados (no solo españoles) fueron detenidos súbitamente y expulsados (sin juicio) a Argelia y Córcega (y partir de ahí a otros países como Checoslovaquia), lejos de sus familias. El contexto de la Guerra Fría aumentó la presión contra todo tipo de colectivos, sospechosos de tener un enlace con la Komintern y la red de espionaje ruso. Los exiliados en Toulouse y, especialmente, la gestión y dirección del hospital de Varsovia, claramente estaba en el punto de mira de esta intervención policial. Piñar publicó su trabajo justo un año antes de la operación Boléro-Paprika, en la que la policía francesa arrestó el personal sanitario del Hospital, acusándoles de ser agentes secretos comunistas (Dreyfus-Armand, 1999; Guixé, 2002, 2012; Martínez Vidal, 2010). La operación policial significó el cierre del hospital Varsovia, que a partir de ese momento pasó a manos de médicos franceses (Martínez Vidal, 2010).

Es posible que Piñar fuese comunista. Su nombre aparece en el listado policial, aunque no hay información sobre su arresto o su expulsión (Guixé, 2002). Como he mencionado antes, es probable que el médico español no se quedara en el hospital, sino que se hiciera cargo de la dirección de la Maison des Enfants de Saint-Goin, una institución situada en un lugar recóndito de los Pirineos. Tal hecho explicaría por qué Piñar no fue expulsado de Francia, conjuntamente con la dirección del Hospital. Con los cambios también desapareció la revista del hos- pital, en la que Piñar había publicado su informe. Los Annales habían nacido con la voluntad de ser un instrumento de divulgación de la obra llevada a cabo en el hospital (Ferran, 2008). El artículo de Piñar sobre el rastro de la guerra en los niños cumplía plenamente con el objetivo que caracterizaba la revista: velar por la salud de la población exiliada y dar a conocer los sufrimientos y las carencias de la población española emigrada.

Pero el mensaje llegó a destiempo. Como muestra la publicación de la UNESCO (Brosse, 1949); en ese momento el Gobierno francés y las organizaciones humanitarias internacionales estaban preocupados por otros colectivos, mayores en número y con situaciones todavía más dramáticas. La Cruz Roja informaba que la guerra había dejado un total de 250.000 huérfanos en el país. Entre ellos destacaban los 40.000 niños judíos. Gran parte había pasado por campos de concentración, la mitad habían perdido ambos progenitores y, en promedio, mostraban un retraso escolar de 2 a 5 años (Brosse, 1949). Tras la posguerra, resultó una situación difícil de afrontar para la Cuarta República Francesa en plena Guerra Fría.

\section{Conclusión}

Con su publicación, el médico español quería llamar la atención sobre la situación de los jóvenes exiliados, un colectivo que en ese momento se encontraba socialmente descontextualizado y marginado (Piñar, 1949). Resultaba importante para él mostrar a través de test psicológicos que estos niños no eran intelectualmente inferiores, aunque según el nivel educativo lo parecían. Asimismo, quería enfatizar el hecho de que ninguno de los niños españoles estudiados tenía antecedentes criminales. Como se ha visto, en la literatura internacional justamente se había destacado una decadencia del carácter de los jóvenes y, con ello, un aumento en la delincuencia infantil como uno de los efectos más notables de la guerra.

Si tenemos en cuenta su investigación y la información sobre las condiciones de vida de los niños exiliados en Francia, creemos que los factores más graves que dificultaban la vida fueron: el recuerdo 
de la guerra expresado en pesadillas, los problemas afectivos debido a la pérdida del hogar familiar (en muchos casos de la figura del padre), la pobreza y el retraso escolar. La publicación del médico español quiso contribuir al conocimiento científico dentro del campo de la psicología infantil, a la vez que tenía la intención política de valorar y pedir ayuda psicológica y material para este colectivo ante la opinión pública y los expertos internacionales.

Una comparación con las publicaciones científicas de la época realizadas con otro tipo de muestras infantiles (generalmente niños ingleses, norteamericanos y franceses) pone en evidencia una correlación entre los resultados obtenidos. Otros investigadores también habían comprobado un efecto de la guerra en el ámbito afectivo, problemas en mantener la atención y un retraso escolar, sin que ello afecte el nivel de inteligencia. Asimismo, las investigaciones con jóvenes franceses comprobaban cierto rastro de las experiencias traumáticas en la memoria y el inconsciente del joven, especialmente visible en los sueños, las actitudes y las reacciones emocionales. Ante la pregunta acerca del tipo de problema psicológico creado por las secuelas, $\mathrm{Pi}$ ñar (1949) parece seguir la línea mayoritaria de los que abogan por un cuadro sintomático y una problemática psíquica normal, similar a cualquier reacción intensa ante una situación de estrés, miedo o angustia. En este sentido, se alejaba del planteamiento adoptado por Wolf-Machoel (1945), quien identificaba tipos específicos de psicosis para los desplazados y los internados. A pesar de ello, ambos coincidieron en exigir una intervención psicológica y psicoterapéutica de los afectados.

Aunque no fuera directamente el caso de Piñar, resulta interesante cómo algunos investigadores de la época como Heuyer (1948) responsabilizaron a la madre de los problemas psicológicos y criminológicos de los jóvenes. El afán personalizador que caracteriza la perspectiva clínica, individual y psicoanalítica adoptada en gran parte de los trabajos, les lleva a focalizar la atención en la figura materna. Por un lado, insistieron en la necesidad de mantener madre e hijo(s) juntos y evitar a toda costa una evacuación de la población. Por el otro, la consideraron incapaz de ofrecerles un entorno psicológico y moral adecuado, supliendo la ausencia de la autoridad paterna. Con ello la acusaron de una decadencia moral y no mencionan otros factores circunstanciales de peso, característicos de los tiempos convulsos de guerra y posguerra, como fueron las agresiones sufridas por las mujeres, la falta de medios y la consecuente necesidad vital de conseguir ingresos. Los informes de la época evidencian la dificultad de mantener una rutina escolar en tiempos de guerra (especialmente cuando se trataba de población desplazada), por lo que muchos niños quedaban desatendidos mientras la madre trabajaba o buscaba medios de sustentación. Además, las encuestas dejaban patente la influencia nociva del ambiente vivido en los campos de refugiados, detectable especialmente en las observaciones de las jóvenes (Wolf-Machoel, 1945).

Sin duda, el diagnóstico y la reflexión sobre la condición psicológica del niño ayudaron a plantear medidas de apoyo terapéutico, así como estrategias pedagógicas para los maestros que tenían que tratar con un colectivo difícil. Estos debían educar a jóvenes que habían quedado afectados anímicamente por las experiencias vividas. Más que en otras generaciones, hubo entre ellos los que habían madurado sexualmente de forma prematura, los que sabían de la doble moral de los adultos y retaban su autoridad. Asimismo, tanto el estudio de los exiliados españoles, como otras investigaciones psicológicas sobre las secuelas de la guerra, crearon una situación que permitía al joven reflexionar y comunicar sus experiencias. Se trataba de una actividad que, en ocasiones, pudo resultar reconfortante para los participantes.

Aun así, el intento del médico español de ayudar a los jóvenes más bien resultó un fracaso. Históricamente la publicación fue lanzada en un mal momento. El intento de llamar la atención de la comunidad internacional sobre la difícil situación de los jóvenes exiliados en Francia, enseguida quedaría sepultado ante el nuevo escenario de la Guerra Fría. En el marco de la colaboración entre Francia y la España de Franco, tuvo lugar un ataque policial que no solo impidió una atención terapéutica a los jóvenes, sino que terminó con el apoyo clínico y social que instituciones como el Hospital de Varsovia y la Maison 
des Enfants de Saint-Goin estaban prestando a la comunidad exiliada. Debido a la coyuntura política del momento, la publicación no alcanzó su objetivo político. Además, al estar publicado en una revista de una tirada limitada y cuyos números no fueron vendidos sino distribuidos entre emigrantes y benefactores (Pons, 2010), tampoco obtuvo ningún impacto científico.

A pesar de ello, y por presentar una visión única del estado mental y anímico de un sector joven, perjudicado por la situación social y política del momento, pienso que el trabajo de Piñar (1949) tiene suficiente valor como para ser conocido más ampliamente.

\section{Referencias}

Alcock, A. T. (1941). The bombed child and the Rorschach test. British Medical Journal, 2(4221), 787.

Aldecoa, J. (1983). Los niños de la guerra. Madrid: Anaya. Alted, A. (1996). Las consecuencias de la Guerra Civil española en los niños de la República: de la dispersión al exilio. Espacio, Tiempo y Forma, 9, 207.

Alted, A., \& Domergue, L. (Eds.). (2003). El exilio republicano español en Toulouse, 1939-1999. Madrid: UNED.

Barona, J. L. (2004). El Consejo Superior de Protección a la Infancia y Represión de la Mendicidad (1904-1914). Su ideología social y sanitaria. En E. Perdiguero (Comp.), Salvad al niño: estudios sobre la protección a la infancia en la Europa mediterránea a comienzos del siglo XX (pp. 121-154). Valencia: Guada.

Barona, J. L. (Ed.). (2010). El exilio científico republicano. Valencia: Universitat de València.

Benjamin, N. (Ed.). (2007). Recuerdos: The Basque children refugees in Great Britain. Norwich: Mousehold Press.

Beverly, B. (1943). Effect of war upon the minds of children. American Journal of Public Health Nations Health, 33(7), 793-798.

Bodman, F. (1944). Child psychiatry in war-time Britain. Journal of Educational Psychology, 35(5), 293-301.

Bravo, B., Latorre, M., Romero, C., Sarmiento, L., Alonso, B., Tarragona, M., ... Pons, G. (1993). Nuevas raíces: testimonios de mujeres españolas en el exilio. Barcelona: Planeta.

Brosse, T. (1949). L'enfance victime de la guerre: une étude de la situation européenne. París : UNESCO.

Cervera Gil, J. (2007). La guerra no ha terminado: el exilio español en Francia (1944-1953). Madrid: Taurus.

Cuesta, J., \& Bermejo, B. (Eds.). (1996). Emigración y exilio: españoles en Francia. Madrid: Eudema.

Despert, L. (1944). Effects of war on children's mental health. Journal of Consulting Psychology, 8(4), 206-217.

Dreyfus-Armand, G. (1999). El exilio de los republicanos españoles en Francia: de la Guerra Civil a la muerte de Franco. Barcelona: Crítica.

Duroux, R., Baudon, G., \& Gaboardi, L. (2006). Lo que yo he visto en la guerra: los dibujos infantiles de la colección Brauner 1937-1938. Guadalajara: Diputación Provincial de Guadalajara, Servicio de Cultura.

Ferrán Sánchez, A. (2008). Medicina a l'exili de Toulouse: Annals de l'Hospital Varsòvia, Gimbernat, $50,331-347$.

Fondation Nationale des Sciences Politiques (Centre d'Histoire de l'Europe du Vingtième Siècle). (1999). Enfants de la guerre civile espagnole: vécus et représentations de la génération née entre 1925 et 1940. París: L'Harmattan.

Freud, A., \& Burlingham, D. (1942). Young children in war-time: A year's work in a residential war nursery. London: G. Allen y Unwin.

Gallardo J. A., \& Gallardo, M. E. (2011). La música dibujada por niños durante la Guerra Civil española. Música y Educación, 24(85), 78-105.

Gerard, M. (1943). Psychology of pre-adolescent children in war time: 1 . Psychological effects of war on the small child and mother. American Journal of Orthopsychiatry, 13(3), 493-496.

Ginestá, J. M. (2010). Los republicanos españoles en la Francia de 1939. La experiencia del exilio. En F. Durán \& C. Ruiz Barrientos (Eds.), La España perdida: los exiliados de la II República (pp. 361-376). Córdoba: Universidad de Córdoba.

Giral, F. (1994). Ciencia española en el exilio (19391989): el exilio de los científicos españoles. Barcelona: Anthropos.

Glondys, O. (2012). La guerra fría cultural y el exilio republicano español. Madrid: CSIC. 
Guerra, F. (2003). La medicina en el exilio republicano. Madrid: Universidad de Alcalà.

Guixé Coromines, J. (2002). L'Europa de Franco: L'esquerra antifranquista i la «caça de bruixes» a l'inici de la guerra freda, França 1943-1951. Barcelona: Publicacions de l'Abadia de Montserrat, VEGAP.

Guixé Corominas, J. (2010). Solidaritat humana i resistència política sota control policial: L'Hospital de Varsòvia en el marc de la Guerra Freda (1944. 1950). En À. Martínez Vidal (Coord.), Exili, medicina i filantropia: L'Hospital de Varsòvia de Tolosa de Llenguadoc (1944-1950) (pp. 51-70). Barcelona: Afers.

Guixé Corominas, J. (2012). La República perseguida: exilio y represión en la Francia de Franco (1937-1951). Valencia: Universitat de València.

Herrero, F. (2003). Mercedes Rodrigo: una pionera de psicología aplicada en España y Colombia (Tesis Doctoral). Facultad de Filosofía, Universidad Complutense de Madrid.

Heuyer, G. (1948). Psychopathologie de l'enfance victime de la guerre. Sauvegarde. Revue des Associations Régionales de Sauvegarde de l'Enfance et de l'Adolescence, 17, 3-43.

Jersild, A. T., \& Meigs, M. F. (1943). Children and war. Psychological Bulletin, 40(8), 541-573.

Jouhy, E., \& Shentoub, V. (1949). L'évolution de la mentalité de l'enfant pendant la guerre. Neuchâtel: Delachaux et Niestlé.

Kimmins, C. W. (1915). The special interests of children in the war at different ages. Journal of Experimental Pedagogy and Training College Record, 3, 145-152.

Kirchner, M. (1974). La psicología aplicada en Barcelona (1916-1936) (Tesis Doctoral). Facultad de Filosofía y Ciencias de la Educación, Universidad de Barcelona.

Langdon-Davies, J. (1938). Air raid. London: Routledge. Lézine, I. (1948). L'enfant et la guerre. Enfance, 1(2), 142-158.

Mac Donald, M. (1943). Impact of war on children and youth-intensification of emotional problems. American Journal of Public Health Nations Health, 33(4), 336-338.

Marqués, P. (1993). Les enfants espagnols réfugiés en France (1936-1939). París: Autor.
Martín, J., \& Carvajal, P. (2002). El exilio español (19361978). Barcelona: Planeta.

Martínez Vidal, À. (Coord.). (2010). Exili, medicina $i$ filantropia: l'Hospital Varsòvia de Tolosa de Llenguadoc (1944-1950). Catarroja: Afers.

Mercier, M. H., \& Despert, L. (1943). Psychological effects of the war on French children. Psychosomatic Medicine, 5(3), 266-272.

Midgley, N. (2007). Anna Freud: The Hamstead War Nurseries and the role of direct observation of children for psychoanalysis. International Journal of Psychoanalysis, 88, 939-959.

Mira y López, E. (1939). Psychiatric experience in the Spanish War. British Medical Journal, 4093, 12171220.

Mülberger, A. (2013). "Ells necssiten ser tractats psicoterapèuticament": l'impacte psicològic de la guerra entre els infants espanyols acollits a França. En R. Barrié, M. Camiade \& J. Font (Eds.), Actes du 2d Séminaire Transfrontalier. Déplacements forcés et exils au XXe siècle-Le corps et l'esprit (pp. 149-168). Perpinyà: Talaia.

Nieto, A. (2007). Las guerrillas antifranquistas (19361965). JC Clementine.

Pàmies, T. (1977). Los niños de la guerra. Barcelona: Bruguera.

Perdiguero, E. (Ed.). (2004). Salvad al niño: estudios sobre la protección a la infancia en la Europa mediterránea a comienzos del siglo XX. Valencia: Guada.

Perdiguero, E., \& Robles, E. (2004). La protección a la infancia y la Sociedad Española de Higiene. En E. Perdiguero (Ed.), Salvad al niño: Estudios sobre la protección a la infancia en la Europa mediterránea a comienzos del siglo XX (pp. 93-120). Valencia: Guada.

Piñar, A. (1949). Las huellas de la guerra en el psiquismo infantil y juvenil. Anales del Hospital Varsovia, 2(3), 1-3.

Pons Barrachina, A. (2010). Anales del Hospital Varsovia: mirall, mirador i mostrador. En À. Martínez Vidal (Coord.), Exili, medicina i filantropia: L'Hospital Varsòvia de Tolosa de Llenguadoc (1944-1950) (p. 71). Catarroja: Afers.

Preston, R. C. (1942). Children's reactions to the contemporary war situation (Child Developmental Monograph № 28). Columbia: Columbia University. 
Rafaneau-Boj, M. C. (2010). Los campos de concentración de los refugiados españoles en Francia. En F. Durán \& C. Ruiz Barrientos (Eds.), La España perdida: Los exiliados de la II República (pp. 315-344). Córdoba: Universidad de Córdoba.

Rautman, A., \& Brower, E. (1951). War themes in children's stories, six years later. The Journal of Psychology, 31, 263-270.

Rollet, C. (2001). Les enfants au XIXe siècle. París: Hachette.

Ross, H. (1943). Psychology of pre-adolescent children in war time. III. Emotional forces in children as influenced by current events. American Journal of Orthopsychiatry, 13(3), 502-504.

Sierra, V. (2009). Palabras huérfanas: los niños de la Guerra Civil. Madrid: Taurus Historia.

Stückelberger, A. (1943). Der Einfluss des Kriegsgeschehens auf das Geistesleben des Schulkindes. [La in- fluencia de la guerra en la mente de los escolares] Oxford: Gotthelf Verlag.

Wolf-Machoel, J. (1945). La réadaptation de la jeunesse et des déracinés de guerre. Boudty, Suiza: Éditions de la Baconnière.

Young, F. M. (1945). A juvenile case of war-connected trauma. The Journal of Psychology, 19, 31-42.

Young, F. M. (1947). Psychological effects of war on young children. American Journal of Orthopediatry, $17,500-510$.

Zahra, T. (2009). Lost children: Displacement, family, and nation in postwar Europe. The Journal of Modern History, 81(1), 45-86.

Zarzoso, A., \& Martínez Vidal, À. (Eds.). (2011). Medicina, guerra i exili: una generació destruïda per la guerra. Barcelona: CSIC. 
